\title{
NEOLIBERALISMO Y DISCURSOS PRESIDENCIALES EN COSTA RICA 1998-20021
}

\section{NEOLIBERALISM AND PRESIDENTIAL DISCOURSES IN COSTA RICA 1998-2002}

\section{Chester Urbina Gaitán *}

RESUMEN

Los discursos presidenciales del ex Presidente Miguel Ángel Rodríguez (1998-2002) exponen como la teoría económica fue usada como soporte ideológico para insertar a Costa Rica en la globalización económica.

PALABRAS CLAVE: COSTA RICA * NEOLIBERALISMO * GLOBALIZACION * DISCURSO * PRESIDENTE $*$ ESTADO

ABSTRACT

Presidential discourses of ex President Miguel Angel Rodriguez (1998-2002) exposed as economic theory was used as ideological support for inserting Costa Rica in economic globalization.

KEYWORDS: COSTA RICA * NEOLIBERALISM * GLOBALIZATION * DISCOURSE* PRESIDENT * STATE

$1 \quad$ El autor agradece los comentarios a una versión preliminar del Dr. Guillermo Carvajal Alvarado.

* Centro de Investigación y Conservación del Patrimonio Cultural, Costa Rica.

chesterurbina@yahoo.com 


\section{INTRODUCCIÓN}

El estudio de los discursos presidenciales en Costa Rica, permite reconocer como la clase dominante ha ido estructurando a lo largo del tiempo su visión política de sociedad, la cual muchas veces se adapta a los intereses personales o de fracción política de algunos gobernantes, en vez de enfocarse en lo que la sociedad nacional necesita para su sobrevivencia y reproducción. Acuña en su trabajo pionero: "Historia del vocabulario político de Costa Rica. Estado, república, nación y democracia (1821-1949)", concluye que hasta en la fundación de la República de Costa Rica en 1848, la Nación por inventarse era la centroamericana y no la costarricense. Después de ese año, se trasfiere esa tarea a Costa Rica, pero bajo el concepto político de nación. Hasta la primera década del siglo $\mathrm{xx}$, persistió en el vocabulario político la aspiración de reconstruir la nación centroamericana, meta que desaparece de manera definitiva a inicios de la década de 1920 (Taracena y Piel, 1995: 63-74). En el corpus analizado por Acuña, no es fácil discernir el contenido cultural del nacionalismo oficial costarricense. No hay ningún discurso que pretenda llevar la existencia de la nacionalidad costarricense hacia la época colonial o hacia el fondo de los tiempos.

El comercio del café dará a la clase dominante la base económica para la existencia del Estado costarricense y la viabilidad de un proyecto de nación de corte liberal, donde el rescate y reelaboración de la Campaña Nacional de 1856-1857, desempeñó un papel de primer orden (Molina y Palmer, 1992: 169-205; Taracena y Piel, 1995: 75-85). Tal situación permite confirmar que la estructura de una sociedad en un lugar y momento dados, así como, las ideologías prevalecientes, se determinan en gran parte por la manera en como dicha sociedad -mejor dicho la clase dominante - afronta $y$ resuelve sus necesidades de orden material (Carr, 1969: 32).

Jara ha apuntado que en el último discurso presidencial de Rodríguez Echeverría, los principales tópicos de su proclama fueron: el trabajo de los costarricenses, necesidades para el futuro: educación e infraestructura, telecomunicaciones, reducción de tasas de interés $y$ de inflación, reforma fiscal, acceso al crédito, reforma laboral, aprobación del Tratado de Libre Comercio con Estados Unidos, así como, aportes de gobiernos anteriores y el "Triángulo de la Solidaridad" (2006: 141-183). Pese a este aporte, no se analiza el tipo de estructura social que este ex mandatario proponía, por lo que este artículo tiene como objetivo realizar un análisis crítico de los discursos presidenciales del ex presidente de la República Dr. Miguel Ángel Rodríguez Echeverría (1998-2002), enfatizando en la forma en que la teoría económica neoliberal se presenta como la forma de inserción del país a la globalización económica.

\section{LIBRE MERCADO, EDUCACIÓN, TRABAJO Y LOS DISCURSOS PRESIDENCIALES DE COSTA RICA 1998-2002}

Según Cuevas, en el campo cultural en Costa Rica, el Estado ha sido el principal dinamizador de la vida cultural, abriendo espacios y creando instituciones donde diferentes sectores de la población han encontrado posibilidades de expresión. Su fuerte presencia, ha sido también la que ha marcado los más importantes límites al campo cultural costarricense. Por ser este un Estado con alto nivel de legitimidad (que se logra también en el ámbito de la cultura), sus criterios son esenciales para la sanción social respecto a lo que produce el campo cultural (Cuevas, 1996: 176). Pese a que el Estado no ha perdido su papel de director intelectual de la sociedad, de una forma paulatina después de 1990, las políticas culturales se han despolitizado, en tanto que la vinculación colectiva gira en torno a lo privatizado, lo desregulado y lo mercantilizado.

Con respecto a los discursos presidenciales de los gobiernos anteriores al del Dr. Rodríguez Echeverría, se tiene que durante el mandato de Rafael Ángel Calderón Fournier se utilizó la frase: "Costa Rica se supera", con la que se pretendía justificar y valorar la necesidad de introducirse en el contexto de globalización, que era rechazado por los sectores económicos más conservadores (Mena y Soto, 1997: 132). Por su parte, José María Figueres Olsen apeló al Espíritu de superación, lucha y solidaridad que caracterizan al Ser Costarricense y que según 
él, han llevado al país a apropiarse de estándares comparables a los que usualmente poseen los países desarrollados en el campo social y económico. Los valores nacionales fueron utilizados como elemento decorativo y legitimante dentro del discurso presidencial (Mena y Soto, 1997: 146).

En su mensaje de toma de posesión del 8 de mayo de 1998, el Dr. Miguel Ángel Rodríguez Echeverría comienza proclamando lo siguiente:

Hechos a imagen de Dios - mujeres y hombres - fuimos instruidos desde el propio Génesis, cuando empezamos a habitar la tierra, por el más antiguo de los mandamientos: "CRECED...", Este fue el primer imperativo divino que recibió la humanidad: "CRECED..." Con esta orden profunda en nuestros corazones la humanidad ha crecido durante milenios, en número y en capacidad, en conocimientos $y$ en tradiciones, en valores, libertad, igualdad $y$ hermandad. CREANDO, CRECIENDO hacia ese inagotable mañana que avizoramos pleno de esperanza (Mena, 2002: 473).

Las referencias bíblicas son parte del pensamiento de Rodríguez. En este discurso se encuentra una posición a la que Michael W. Apple ha tildado de neoconservadora, ya que en gran medida, aunque no del todo, se basa en una valoración romántica del pasado en el que imperaban "el verdadero conocimiento" y la moralidad, donde cada persona "conocía su lugar" y unas comunidades estables guiadas por un orden natural, nos protegían de los males de la sociedad (Apple, 2002: 65).

Según Martínez, el mercado es el modo en que la sociedad ("avanzada", postmoderna) se organiza, mientras el ser humano se halla en niveles inferiores de la conciencia (el nivel mental ya sea mítico o racional) (2009: 34-56). Muchos economistas de escuelas heterodoxas, han resaltado el carácter religioso de la ciencia económica. Inclusive los economistas ortodoxos afirman que los dos sistemas históricos más importantes configuradores del ser humano son la religión y la economía (Marshall, 2005).
Para Petrella, el "evangelio de la competitividad" cuenta con evangelistas y teólogos. Los evangelistas son los economistas - como el ex presidente Rodríguez Echeverría- y expertos que han codificado la economía de mercado con base en pensadores y científicos como Hobbes, Darwin, Spencer y Nietzsche, a quienes se apela siempre que sea necesario. Los teólogos son los ideólogos que tratan de demostrar que la competitividad afecta a todos los ámbitos de la vida y es la única tabla de salvación: "es como la gracia. Se tiene o no se tiene. No es divisible. Aquellos que la tienen se salvaran. Aquellos que cometan el pecado de no ser competitivos están condenados a desaparecer" (Petrella, 2003-2004).

Esto se comprueba cuando el ex mandatario señala:

... la necesidad de la competencia como mecanismo que estructura los mercados se encuentra en primer lugar dentro de nuestras prioridades. Para ello es esencial, en particular para los países pequeños, la apertura comercial. El imperativo de la competencia es una fuerza al servicio de la creación. La protección es cosa buena para los niños pero el joven necesita aumentar su autonomía para vivir su propia vida. Así como el joven necesita de la libertad para encontrar su camino, así también el país necesita de la competencia y la apertura para descubrir sus fortalezas y llevarlas a su máximo potencial (Mena, 2002: 477).

Bajo la influencia de la teoría neoliberal, el capital y la riqueza se distribuyen entre los sectores más acomodados, mientras las virtudes cívicas se subordinan a las directrices del libre mercado como modelo para organizar todos los aspectos de la vida (Henwood, 2003). Los fundadores del pensamiento neoliberal -Hayek, Buchanan, Tullock y Friedmantomaron el ideal político de la dignidad y de la libertad individual como pilar fundamental, que consideraron "los valores centrales de la civilización". En su opinión, estos valores son amenazados por todas las formas de intervencionismo estatal, las cuales sustituyen con valoraciones colectivas sobre la libertad de 
elección de los individuos (Harvey, 2007: 11; Hayeck, 2002; Buchanan y Tullock, 1980 y Friedman, 1966).

Es notoria la existencia de lo que se ha llamado la visión "mecanicista" de la globalización. Esta "falacia" (Pucciarelli, 2002) expone a la globalización como un fenómeno "natural" y afirma que si nos atrevemos a actuar de manera alternativa a sus estipulaciones, sobrevendría una catástrofe económica y social de graves consecuencias (Coraggio, 1999: 99-105 y Aranskind, 2001: 219-244).

Don Miguel Ángel agrega que el Estado y el mercado complementándose, la producción basada en el conocimiento, las economías abiertas y competitivas, son las nuevas realidades que deben ser nuestra oportunidad para el desarrollo humano de todas y de todos (Mena, 2002: 477).

Encontramos en este primer discurso, la influencia del pensamiento de Francis Fukuyama (1990) quien exponía que el capitalismo neoliberal ha triunfado y que, de aquí en adelante, es el paradigma según el cual el mundo, particularmente, los Estados $y$ por ende, las sociedades, entienden, estructuran, ordenan y desarrollan las dinámicas propias de la cotidianeidad.

Posteriormente, el mandatario acota que la economía del país necesita revitalizarse para continuar por el camino del crecimiento. Limitaremos el gasto público, estimularemos la competencia y la apertura económica, lucharemos incansablemente para mejorar la calidad de los servicios públicos que se ofrecen a los costarricenses, así como enfrentaremos el reto del desempleo y del alto costo de la vida, y la ausencia de oportunidades para los jóvenes (Mena, 2002: 481).

También apunta que la capacitación técnica de los trabajadores costarricenses es otro de los grandes retos de este fin de siglo. Se necesita una educación centrada en la producción y capacitada en las nuevas tecnologías que demanda la economía del conocimiento (Mena, 2002: 483).

Sobre estas opiniones, se tiene que ante el rechazo de las directrices keynesianas donde el Estado ve limitado su campo de acción, se corre el riesgo de que los individuos se queden sin representación política, lo cual se manifiesta en la apatía, la confusión y la desorientación. Esto podría llevar a que la política se despersonalice y la sociedad tienda a hablar más de economía, salud, educación, seguridad pública, medio ambiente y familia, es decir, de contenidos más pragmáticos (Brunner, 1994: 93). Asimismo, se corre el riesgo de ver a las instituciones docentes como necesarias y valiosas, en la medida en que ofrecen una formación con probabilidades de ser demandada por el mercado o de conseguir un puesto de trabajo en el sector privado (Torres, 2007: 33).

El líder político costarricense proclama la modernización y el crecimiento del país, así como los beneficios derivados del nuevo orden global en expansión. Sin embargo, no señala el impacto negativo generado por este proceso de apertura económica sobre el déficit comercial en un país periférico como Costa Rica. El abrir en una forma indiscriminada la economía nacional al capital transnacional, se incentivó un fuerte incremento de las importaciones, especialmente de bienes de capital y en menor medida de bienes de consumo, al tiempo que se reduce el incentivo a las exportaciones. De esta manera, en ausencia de una industria manufacturera con capacidad exportadora como la de los países centrales, se impulsaría en el país un creciente déficit comercial, al tiempo que se potenciaba la destrucción de la industria nacional existente.

Por otro lado don Miguel Ángel Rodríguez en el apartado "La capacidad de emprender", resalta que el desarrollo de la microempresa es otro factor complementario que permite emprender esta tarea con miras no solo a las necesidades de los más vulnerables, sino también con la meta de desarrollar las capacidades creativas de las personas y su potencial productivo, para que cada quien pueda valerse por sí mismo (Mena, 2002: 486). Rodríguez es motivado a señalar esto, según lo que Harvey ha señalado, pues dice que de que la idea de libertad "degenera, pues, en una mera defensa de la libertad de empresa" (2007: 44).

Asimismo, el ex presidente es enfático al decir: 
Necesitamos continuar la modernización de la economía y redoblar los esfuerzos para controlar, el costo de la vida. Necesitamos más crecimiento en forma sostenida, más empleos y mejores salarios. Necesitamos disminuir la inflación $y$ reducir la pobreza. No podemos mirar hacia atrás y convertirnos en estatua de sal. Necesitamos mirar hacia adelante, hacia el futuro. En frente está el siglo XXI... el tercer milenio. Necesitamos avanzar a paso firme, para subirnos de una vez y para siempre al tren del progreso y del bienestar para todos (Mena, 2002: 507).

Es notable como en este texto, se manifiesta un rompimiento con el modelo de desarrollo económico articulado a partir de 1948, lo que sustenta la tesis de que la llamada evolución histórica reposa, en general, en el hecho de que la última forma considera a las pasadas como otras tantas etapas hacia ella misma (Marx, 1973: 27).

Para el año 2000, resalta el hecho de que como nación, sabemos que necesitamos cambios profundos, pero todavía no nos ponemos de acuerdo sobre cuáles deben ser esos cambios. Sabemos que las recetas del pasado ya no funcionan... (Mena, 2002: 517).

Además, apunta: "esas mujeres y esos hombres que todos los días luchan por su superación personal y el bienestar de su familia, van a sacar adelante este país" (Mena, 2002: 574). Para legitimar su política económica, el presidente Rodríguez retoma un discurso, el cual Villena ha llamado para el caso del fútbol "la economía moral del sacrificio", donde los sectores conservadores consideran que la sociedad costarricense está atravesando por una "crisis de valores", por una etapa de dramática pérdida de valores $y$ de sentimiento patriótico. Según este discurso, que se puede escuchar cotidianamente en los medios de comunicación, en Costa Rica — una suerte de crisis perpetualos costarricenses estarían abandonando los míticos valores campesinos y serían cada vez menos sacrificados por la patria y solidarios entre sí (Villena, 2006: 136).
En su último discurso presidencial, pronunciado el 1 de mayo de 2002, resalta el protagonismo histórico de estas personas trabajadoras:

... todas y todos ellos ayudaron a llevar adelante un nuevo modelo de desarrollo. Un modelo de desarrollo que representa lo mejor de nuestra tradición y de nuestras aspiraciones como pueblo, porque se basa en el conocimiento, en la capacidad $y$ creatividad de las personas para crear oportunidades de superación para todos e impulsar el progreso del país (Mena, 2002: 593).

En este texto, subyace un desentendimiento de la elite costarricense por asumir la dirección intelectual de la sociedad. Para Held, el sistema de mercado es la base de un orden genuinamente legítimo; pues la libertad económica es, en palabras de Friedman, "un requisito esencial de la libertad política". En particular, el mercado puede garantizar la coordinación de las decisiones de productores y los consumidores sin la dirección de una autoridad central; que cada persona pueda buscar sus propios fines con los recursos a su disposición; el desarrollo de una economía compleja sin una elite que proclame saber cómo funciona todo (Held, 1997: 289).

Pese al interés de implantar este modelo de desarrollo neoliberal, la herencia del modelo socialdemócrata en el país es muy diversa. En Costa Rica, las reformas institucionales y económicas no alcanzaron la magnitud y profundidad de las llevadas a cabo en otras naciones de América Latina y el Caribe. De hecho, ocurrió lo que se llama "un empate de fuerzas sociales", lo cual se refiere a la imposibilidad vivida de alcanzar, como algunos quisieran, el retiro total del Estado de la economía y una liberalización y apertura económica más radical. En este proceso de reforma, la sociedad costarricense tomó el camino de la gradualidad (Mora, 2001: 8).

Con respecto al impacto de la implementación de las políticas neoliberales en el discurso sobre la paz social del país, Molina ha resaltado que de 1990 en adelante, este discurso volvió a experimentar una dicotomía 
fundamental, que enfrenta a intelectuales $y$ políticos de izquierda, sindicalistas y otros líderes $y$ organizaciones populares, con los principales medios de comunicación, las cámaras empresariales y los políticos identificados con ellas. Para los primeros, la paz social está amenazada de muerte por las políticas neoliberales; para los segundos, quienes se lanzan a la calle a protestar contra las políticas privatizadoras, al demostrar que no son pacíficos, no merecen siquiera llamarse costarricenses (Molina, 2002).

\section{CONCLUSIÓN}

De los discursos del ex presidente Rodríguez, se evidencia que la globalización económica que proclama, no solo es de la economía, sino que también de los aparatos ideológicos como la educación y la religión, de allí la importancia en los discursos de este al manipular textos bíblicos para transmitir la cultura globalizante o centrar la educación en la formación para el trabajo y la producción.

El neoliberalismo fue propuesto a la población costarricense como una síntesis inevitable y única para sus vidas. El nuevo léxico que emplea como eje central de sus proclamas, pretende la manipulación de masas, de mentes $y$ de comportamientos. Este discurso del poder dominante neoliberal pretende crear una cultura de mercado sin un asidero histórico y un claro proyecto de nación.

Con el análisis hecho de los discursos presidenciales del período 1994-2002, no se pretende ocultar la necesidad del país de insertarse adecuadamente en la globalización económica, sino de evitar que las supuestas bondades del neoliberalismo - libre comercio, trabajo, competitividad, desarrollo, etc. - oculten su carácter de ideología excluyente, carente de preocupación social, ajenas a nuestra naturaleza e identidad.

BIBLIOGRAFÍA

LIBROS

Acuña Ortega, Víctor Hugo. "Historia del vocabulario político de Costa Rica. Estado, república, nación y democracia (1821-1949)". Identidades nacionales y
Estado moderno en Centroamérica. En: Taracena, Arturo y Piel, Jean. San José. EUCR, 1995: 63-74.

Apple, Michael W. Educar "como Dios manda”. Mercados, niveles, religión y desigualdad. Barcelona: Paidós, 2002.

Brunner, Joaquín J. Bienvenidos a la modernidad. Santiago de Chile: Planeta, 1994.

Buchanan, James y Tullock, Gordon. El cálculo del consentimiento. Madrid: Espasa Calpe, 1980.

Buck Morrs, Susan. Thinking past terror: islamism and critical theory on the left. London: Verso, 2003.

Carr, E. H. La nueva sociedad. México DF: Fondo de Cultura Económica, 1969.

Cuevas Molina, Rafael. El punto sobre la "i": políticas culturales en Costa Rica, 19481990. San José: Ministerio de Cultura, Juventud y Deportes, 1996.

Friedman, Milton. Capitalismo y libertad. Editorial Rialp: Madrid, 1966.

Harvey, David. Breve historia del neoliberalismo. Madrid: Ediciones Akal SA, 2007.

Held, David. La democracia y el orden global. Del Estado moderno al gobierno cosmopolita. Barcelona: Paidós, 1997.

Henwood, Doug. After the new economy. New York: The New Press, 2003.

Marshall, A. Principios de Economía. Madrid: Síntesis, 2005.

Marx, Karl. El capital. Crítica de la Economía Política I. México DF: Fondo de Cultura Económica, 1973.

Mena Brenes, Marco Antonio (comp.). Mensajes presidenciales 1982-2002. San José: Imprenta Nacional, 2002.

Molina Jiménez, Iván. Costarricense por dicha. Identidad nacional y cambio cultural en Costa Rica durante los siglos XIX Y XX. San José: EUCR, 2002.

Molina Jiménez, Iván y Palmer, Steven (eds.). Héroes al gusto y libros de moda. Sociedad y cambio cultural en Costa Rica (1750/1900). San José: Plumsock Mesoamerican Studies y Editorial Porvenir, 1992. 
Mora Alfaro, Jorge. Costa Rica. El camino democrático. San José: EUNED, 2001.

Pucciarelli, A. La democracia que tenemos. Declinación económica, decadencia social y degradación politica en la Argentina actual. Buenos Aires: Libros del Rojas, 2002.

Taracena, Arturo y Piel, Jean. Identidades nacionales y Estado moderno en Centroamérica. San José: EUCR, 1995.

Torres, Jurjo. Educación en tiempos de neoliberalismo. Madrid: Ediciones Morata, 2007.

Villena Fiengo, Sergio. Globalización. Siete ensayos heréticos sobre fútbol, identidad y cultura. San José: Ediciones FarbenGrupo Editorial Norma, 2006.

Von Hayek, Friedrich August. Camino de servidumbre. Madrid: Alianza Editorial, 2002.

PERIÓDICOS

Petrella, R. "El evangelio de la competitividad". Le Monde Diplomatique. Ediciones Cybermonde, SL, diciembre 2003-enero 2004.

\section{REVISTAS IMPRESAS}

Aronskind, R. "Globalización en Argentina, o la voluntad soberana de subdesarrollarse". Época 3 (3). 2001: 219-244.

Coraggio, J. L. “¿Es posible pensar alternativas a la política social neoliberal?”. Nueva Sociedad 164. 1999: 99-105.

Martínez Lozano, E. "El hombre sabio y compasivo: una aproximación transpersonal a Jesús de Nazaret".
Journal of Transpersonal Research 1. 2009: 34-56.

Fukuyama, Francis. “El fin de la historia?”. Revista Estudios Públicos 37. Santiago, 1990.

Jara Murillo, Carla Victoria. "Discurso presidencial costarricense: estructura formal y tópica del último mensaje ante los diputados (1902-2002)". Revista de Filología y Lingüística 32 (2). San José. Editorial de la Universidad de Costa Rica, 2006: 141-183.

\section{REVISTA DIGITAL}

Molina Jiménez, Iván. "Paz social e identidad nacional en Costa Rica durante los siglos XIX y XX. Una introducción al problema". Istmo. Revista virtual de Estudios Literarios y Culturales Centroamericanos 11. 2005. En: <http:// collaborations.denison.edu/istmo/n11/ proyectos/paz.html>

TESIS

Mena, Rosibel y Soto, Annette. "Análisis del juego táctico utilizado por José María Figueres Olsen en sus discursos presidenciales de mayo 1994, 1995, 1996 y 1997”. [Tesis en Periodismo]. Escuela de Ciencias de la Comunicación Colectiva. Universidad de Costa Rica, 1997.

Fecha de ingreso: 02/05/2011 Fecha de aprobación: 17/06/2011 
